\title{
FEM analyses of the ITER EC H\&CD torus diamond window unit towards the prototyping activity
}

\author{
G. Aiello ${ }^{1}$, N. Casal ${ }^{2}$, P. Estébanez ${ }^{3}$, V. Gràcia ${ }^{3}$, M. Henderson², A. Meier ${ }^{1}$, G. Saibene ${ }^{3}$, T. Scherer ${ }^{1}$,
}

S. Schreck ${ }^{1}$, D. Strauss ${ }^{1}$, A. Vaccaro ${ }^{2}$

${ }^{1}$ KIT IAM, Hermann-von-Helmholtz-Platz 1, 76344 Eggenstein-Leopoldshafen, Germany

2ITER Organization, Route de Vinon sur Verdon, 13067 St Paul Lez Durance, France

${ }^{3}$ F4E Joint Undertaking, Josep Pla 2, 08019 Barcelona, Spain

\section{Motivation}

Design of the torus window unit ready to start prototyping and testing activity (upcoming window FDR, but not yet frozen design of the systems surrounding the window)
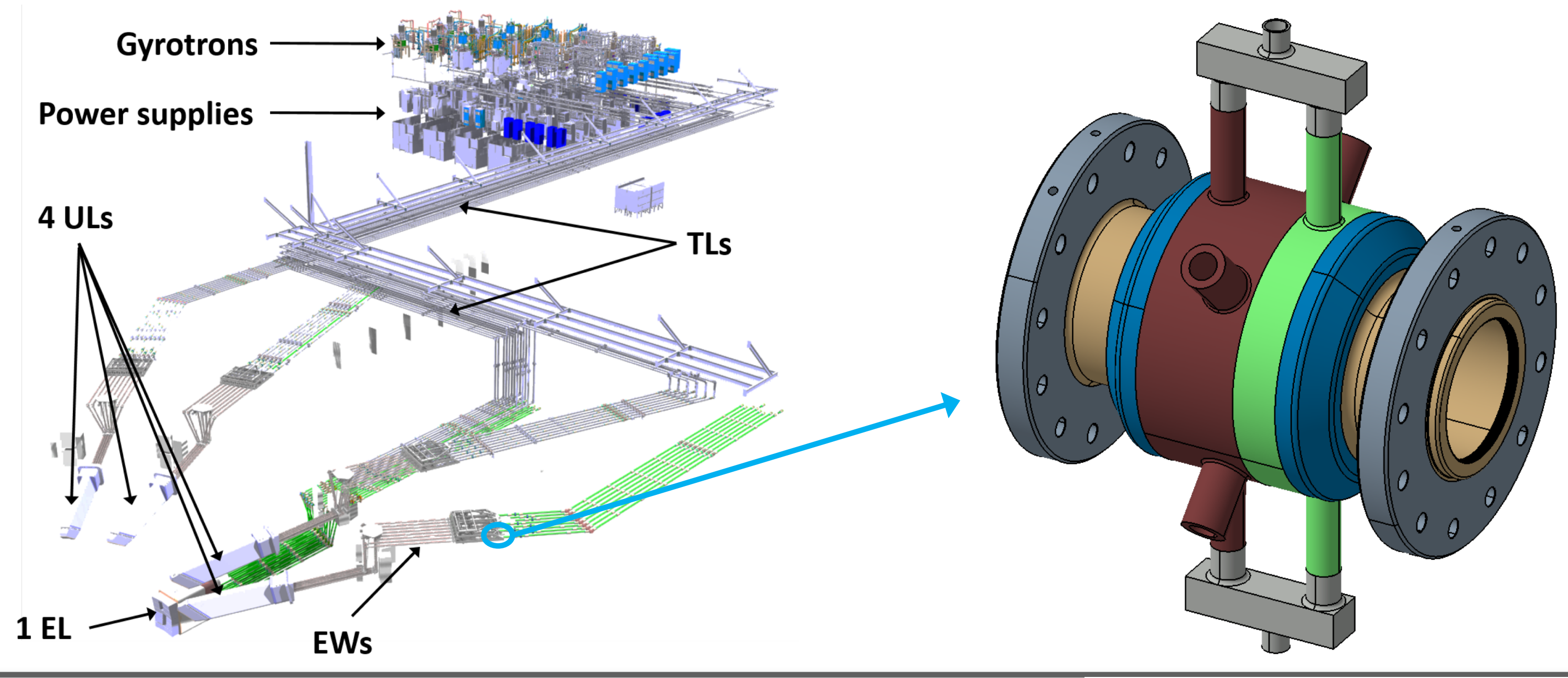

Objectives

- Define requirements for the interface systems of the window in terms of maximum acceptable loads that might be transmitted to the window

- Check the impact of material degradation (due to temperature cycle of brazing process) on the capability of the window outer frame to withstand external loads

- Prove the soundness of the window design before starting the prototyping and testing activity

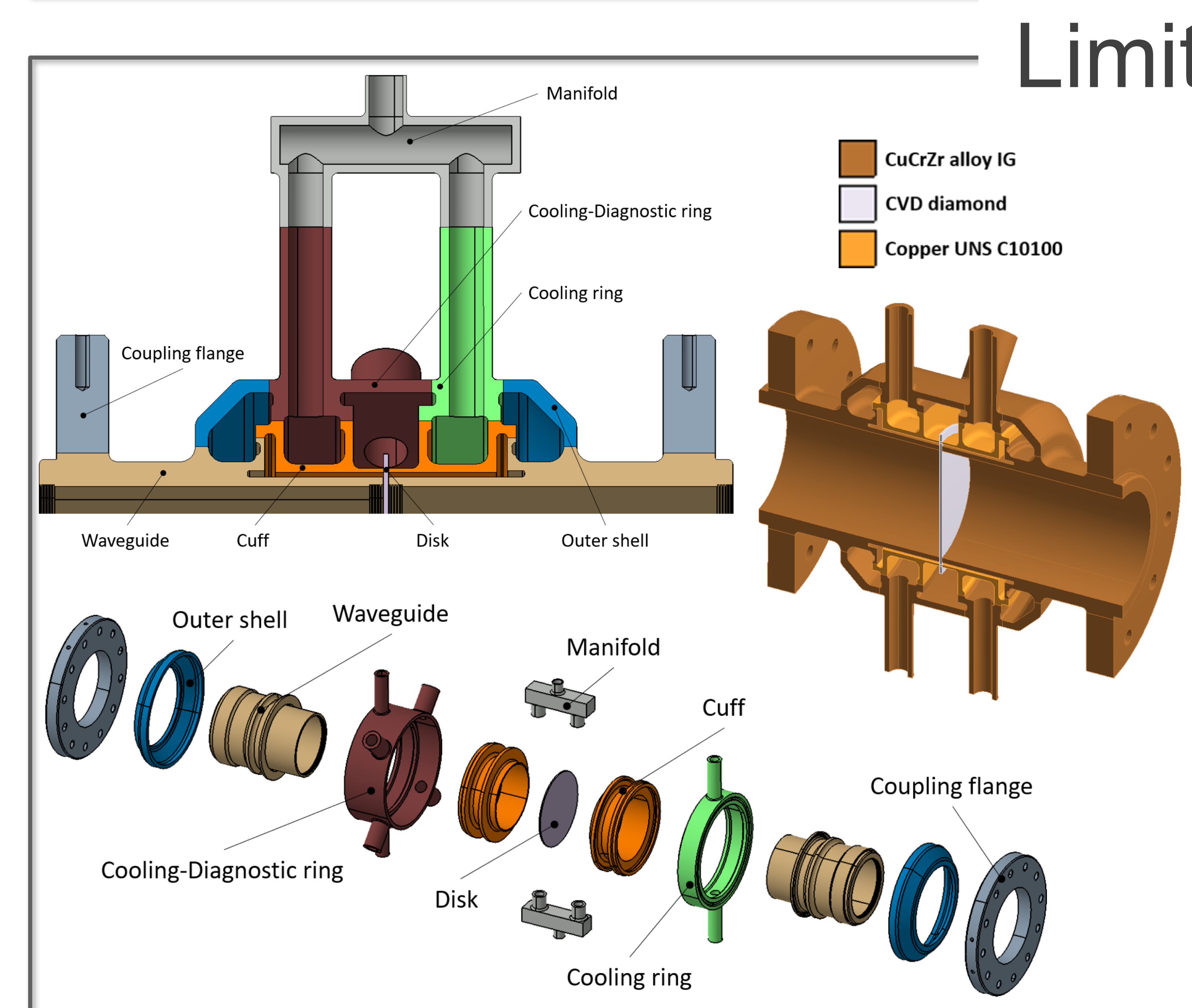

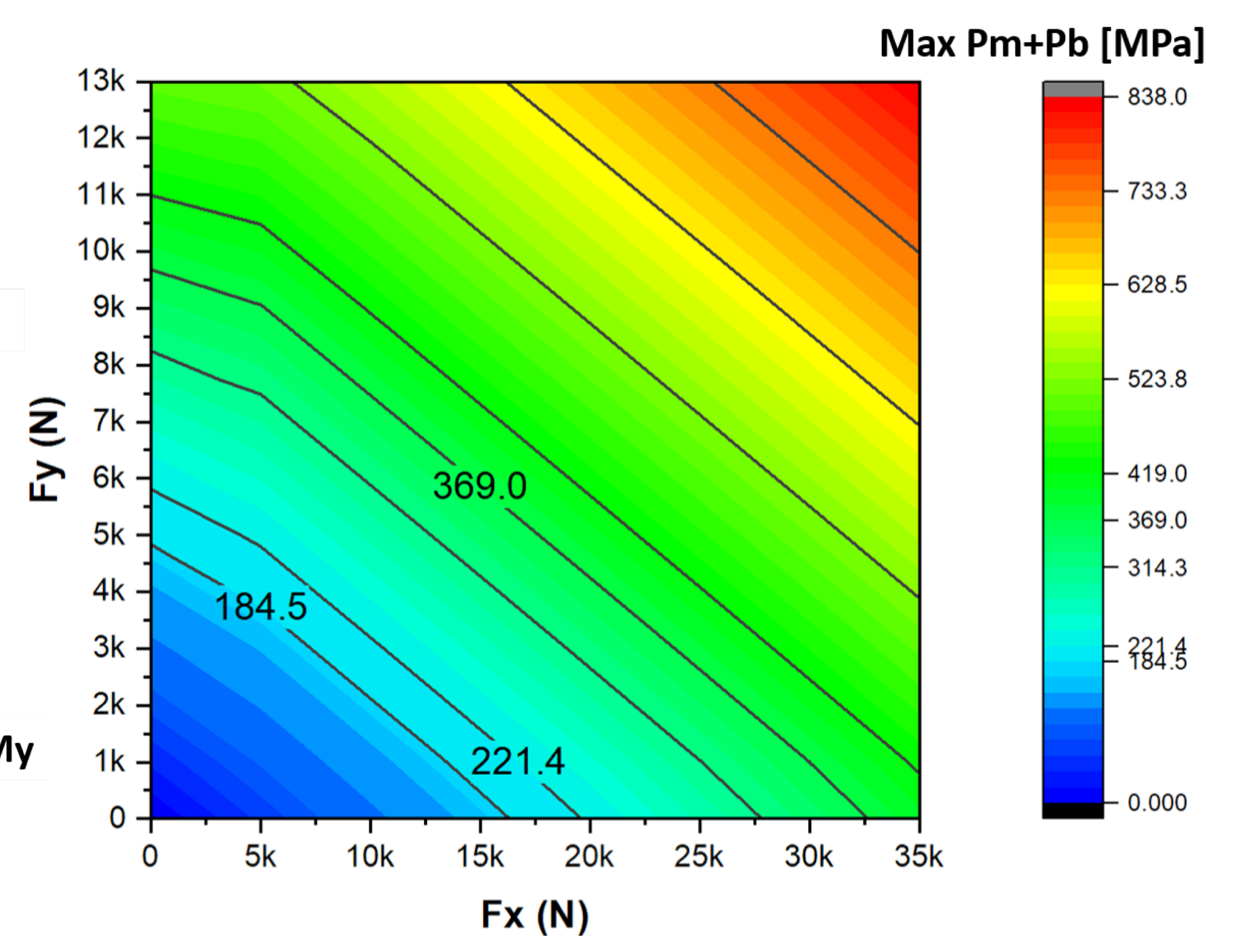

$\mathrm{Fx}(\mathrm{N})$

- A final global analysis of the EWs to provide inputs to the window analysis for the external loads is not currently feasible: not yet frozen design of the EWs

- Limit analysis to find the maximum loads that generate stresses in the window equal to the allowable limits in accordance to: $P_{m} \leq k S_{m}$ and $P_{m}+P_{b} \leq 1.5 k S_{m}$

- Two separate structural analyses with several combinations of forces and moments

- Linearization of resulting stresses and maximum stress components reported in contour plots for both the forces and the moments

- Limit combination of forces and moments for selected design criterion and ASME service level (limit lines in the plots)

- External loads due to both the EWs and TLs systems shall lay in the area of the plots underneath the limit lines of interest

- Total stress due to both forces and moments shall be lower than the allowable limits (generation of grids with stress values from plots)

Example of stress contour plots for the maximum membrane plus bending stress component generated in the window unit in the context of a test run

\section{Analysis strategy}

- Structural analyses with properties of degraded CuCrZr (tensile tests by F4E) to check the impact on window outer frame and optimize design, if required

- Limit analysis to cover all analyses involving the external loads for the window and generate the stress contour plots

- Analyses with load combinations ruled by the limit analysis approach to show that the window design is sound enough for the prototyping: worst case scenarios from limit analyses combined with normal operation (NO), NO plus internal fire, NO plus overpressure

- Analyses for hot spot case and failure of the feeding water system

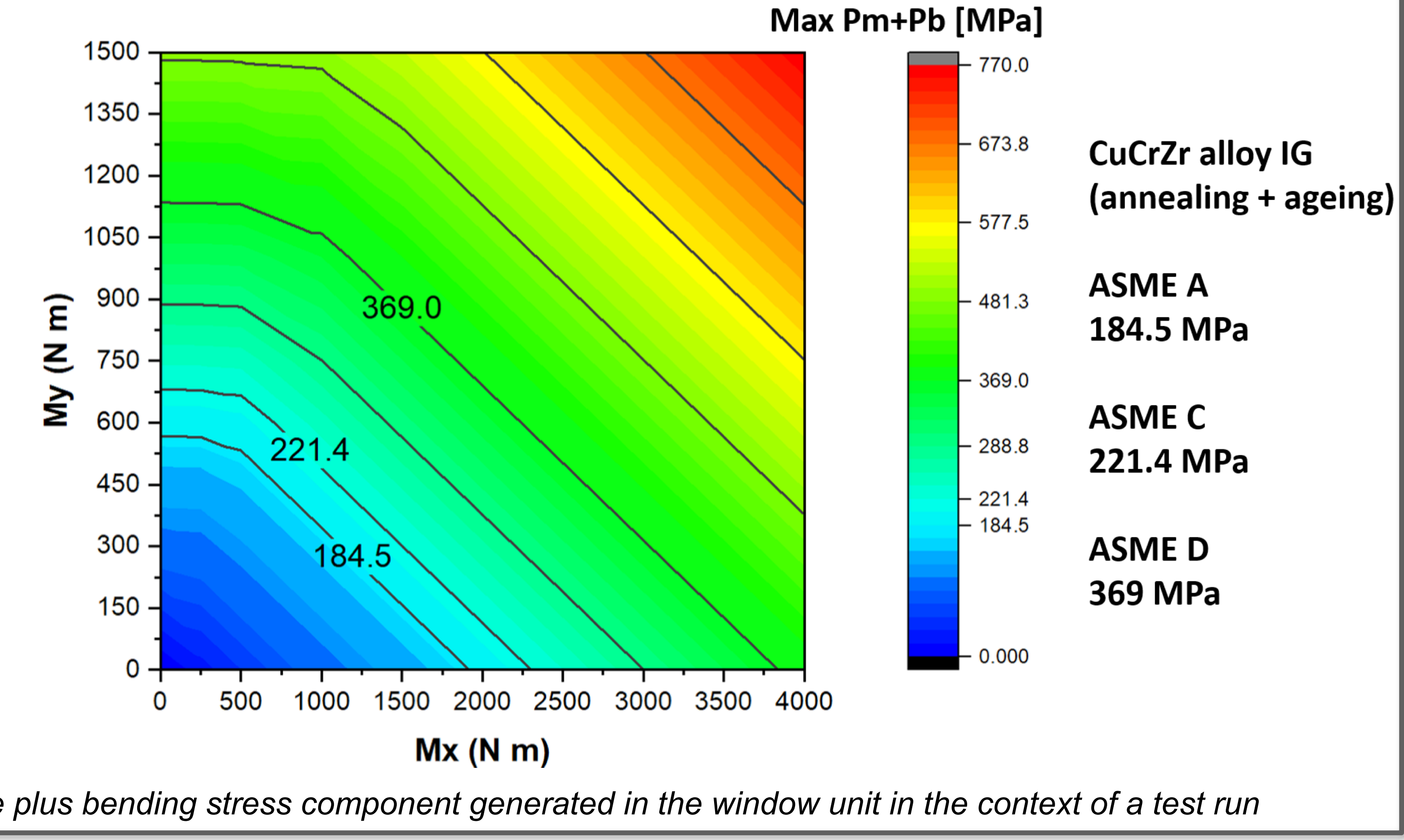

- Prototyping and testing of the window unit - 2020/2021: window Final Design Review (FDR)

- Qualification of the 56 diamond disks and torus window units for the ITER UL and EL plugs

This work was supported by Fusion for Energy (F4E) under the grant contract No. F4E-GRT-615. The views and opinions expressed herein do not necessarily reflect those of Fusion for Energy (F4E) and ITER Organization (IO). F4E and IO are not liable for any use that may be made of the information contained therein. 